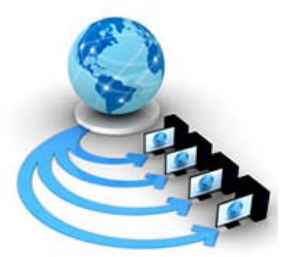

Volume 8, No. 7, July - August 2017

International Journal of Advanced Research in Computer Science

RESEARCH PAPER

\title{
PROPOSING ENHANCED NA GAUN TECHNIQUE (ENGT) FOR RESOURCE BLOCK ALLOCATION IN LTE(LONG TERM EVOLUTION) SYSTEMS FOR IMPROVING QUALITY OF SERVICE
}

\author{
Kawaldeep Kaur and Rimmy Chuchra \\ Department of Computer Science \& Engg \\ Sri Sai College of Engg and Technology, \\ Manawala (Amritsar) Punjab (India)
}

\begin{abstract}
$\overline{\text { Abstract:Due to its distinct and unique features viz. high speed data rate, self-organized network, packet optimized radio access, low latency and }}$ flexible bandwidth deployment LTE (Long Term Evolution) is more preferred in wireless communication. Such kind of terminology uses especially in mobile phones for improving its throughput. LTE uses Orthogonal Frequency Division Multiplexing (OFDM) for the downlink that is, from base station to the terminal to transmit the data over many narrow band carriers each of $180 \mathrm{KHz}$ instead of spreading one signal over the complete $5 \mathrm{MHz}$ career bandwidth. The complete working of LTE is based on the basic principle of OFDMA (Orthogonal Frequency Division Multiplexing). At first step, it divides the broad spectrum into multiple narrow bands and transmits information on this narrow channel in parallel.OFDM meets the LTE requirement for spectrum flexibility and enables cost-efficient solutions for very wide carriers with high peak rates.Orthogonal frequency-division multiplexing (OFDM), is a frequency-division multiplexing (FDM) scheme used as a digital multi-carrier modulation method. Scheduling plays a vital role on the time of resource allocation which has become an essential component for high- speed wireless data systems. This allocation of resources is done according to the need and priority of the user. It has three key components which are involved in the resource allocation and network optimization: resource block scheduling, power control, and client association. The motive to design this new designed methodology is to increase its privacy rate by increasing its security level that automatically improves its speed and data rate. LTE uses OFDM (orthogonal frequency division multiplexing) for downlink transmission. In resource allocation algorithms in OFDM of LTE systems, the allocation of the radio resource has to be in the units of Scheduling Block (SB) and there exists the need for all SBs of each user to use the same Modulation and Coding Scheme (MCS) leading to the degradation of performance. Main challenge for LTE systems is that, by applying OFDMA, multiple resource blocks can be scheduled by a transmission over the frequency and time. In this research paper, we have to focus on resource block scheduling in LTE system and proposes an enhanced version of Na Gaun technique that would be helpful for resource allocation blocks so that user's rate requirement can be fulfilled optimally and throughput of the system can be improved.
\end{abstract}

Keywords:Long term Evolution, wireless communication, throughput, resource allocation techniques,security, scheduling algorithms, modulation and coding schemes and OFDM(Orthogonal Frequency Division Multiplexing).

\section{INTRODUCTION}

Long Term Evolution (LTE)[1] is considered as an advanced tool which is used to improve user's rate requirements, the coverage of high data rates, temporary network deployment, the system throughput and/or to cover new areas [1]. Relaying implies that the terminal communicates with the network via a low-power relay node that is wirelessly connected to a donor cell using the LTE radio-interface technology. From a terminal point of view, the relay node will appear as an ordinary cell. In conjunction with relaying, the terms backhaul link and access link are often used to refer to the eNB(evolved Node B)-relay connection and the relay-UE(User Equipment) connection respectively. By using the backhaul link[2], the cells which are connected to relay node is called as donor cell. Donor Cell which are connected to one or several relays also serve UEs not connected via a relay. Usage of spectrum with respect to the relay node's, operation are classified into inband and outband types.Inband relaying implies that the eNB-relay link shares the same carrier frequency with relayUE links.[11] Outband relaying implies that the backhaul link does not operate in the same carrier frequency as access links. Inband relaying is more flexible in term of allocating resource between backhaul link and access link. A "Type 1 " relay node is an inband relaying node which has the following characteristics. It controls cells, each of which appears to a UE as a separate cell.[8] The relay node shall transmit its own synchronization channels, reference symbols, and so on and the cells may have their Physical Cell ID. In the context of single-cell operation, scheduling information shall receive by UE and HARQ feedback directly from the relay node and send its control channels to the relay node.[6]

For Type 1 relay, due to the relay transmitter causing interference to its own receiver, simultaneous eNB-to-relay and relay-to-UE transmissions on the same frequency resource may not be feasible unless sufficient isolation of the outgoing and incoming signals is provided.[12] In the same way, relay may not be possible to receive UE(user equipment)[8] transmissions simultaneously with the relay transmitting to the eNB. One possibility to handle the interference problem is to operate the relay such that the relay is not transmitting to terminals when it is supposed to receive data from the donor eNodeB (DeNB), i.e. to create 
"gaps" in the relay-to-UE transmission. These "gaps" during which terminals are not supposed to expect any relay transmission can be created by configuring MBSFN (Multicast and Broadcast Single Frequency Network) subframes as exemplified eNB-to-relay transmissions can be facilitated by not allowing any relay-to-UE transmissions in some subframes.[3][10]. These subframes are called backhaul subframes. Subframes in which relay-to-UE transmissions are allowed are called access subframes.
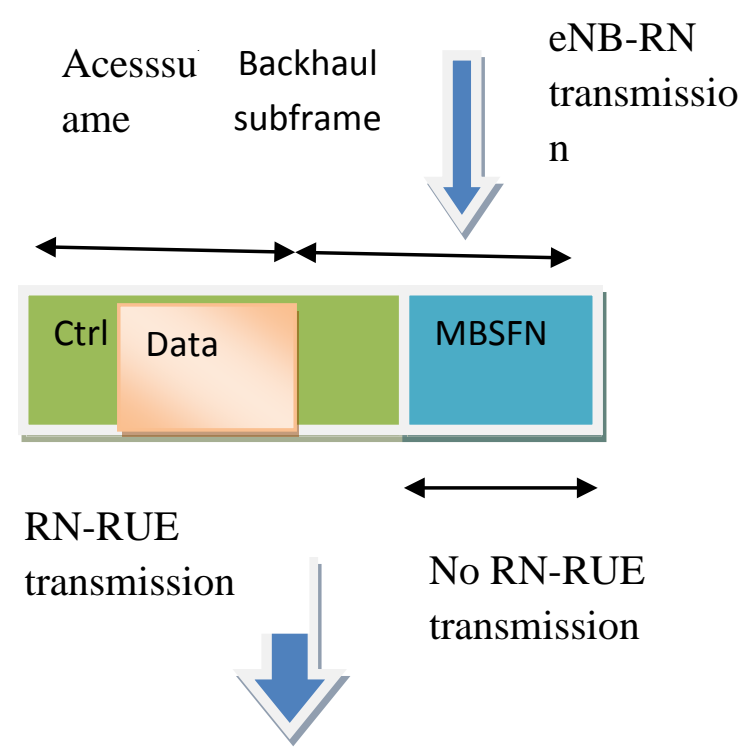

Figure 1: Example of normal subframe and MBSFN subframe at relay node.[8]

In backhaul subframes, relays and macro UEs compete for the precious radio resources in the donor cell for communications. [2][7] How to do the backhaul resource partitioning in an optimized way is very important in the deployment of relays. In this paper we focus on the downlink backhaul resource allocation[4], yet similar approach can be applied to uplink as well.

In this research paper, for proper distribution of resource blocks and achieves maximum throughput authors designed a new methodologyENGTthat is termed asextended Na Gaunn Technique. Resource allocation's role is to dynamically assign available time-frequency resource blocks to different UEs[8] in an efficient way to provide high system performance. In general for resource distribution various algorithms are used viz.First comes first serve (FCFS), Shortest Job First (SJF), Priority scheduling, multi-level scheduling and multilevel feedback queue scheduling etc but the most common scheduling algorithms are round robin scheduling[6], proportional fair algorithm[4] and maximum C/I(channel Indicator) algorithm[7]. The benefit to use this algorithm is these are easy to use as well as easily implemented but correspondingly having some certain drawbacks as like they produces poor throughput results except proportional fair algorithm [2]. This poor throughput[5] ultimately helps to degrade the system performance. So, to overcome this problem of poor throughput [5] \& increase system performance authors work on ENGT.The main objective of this research paper is to provide uniform allocation of resources by its priority and after that finding its maximum throughput rate. The main benefit to utilize this LTE technology [1] is improving its data rate at downlink transmission rate by using OFDMA [9] principle in LTE[1] systems.

\section{RESEARCH DESIGN}

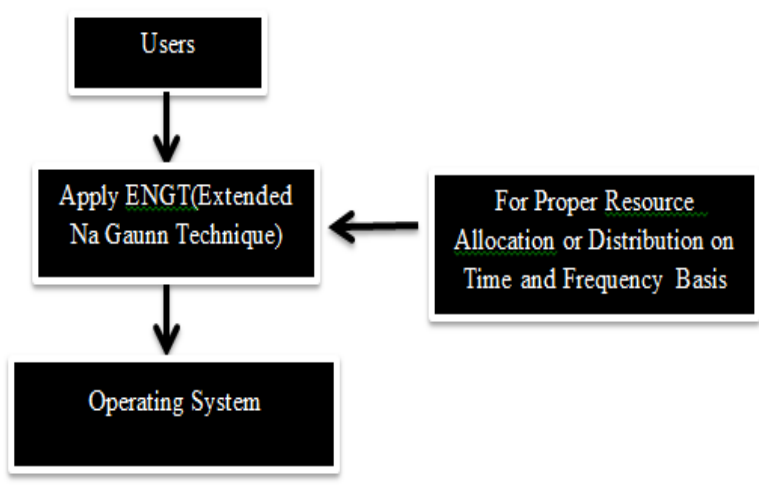

Figure.2: Interaction between User and Operating System.

\section{PROPOSED METHODOLOGY (ENGT- EXTENDED NA GAUNN TECHNIQUE)}

This new designed methodology involves main two steps that can be described below:-

Step-1) Find average channel gain and minimum required SBs (segment block).

Step-2) Prioritize Users.

Step-3) Allocate SB’s only to prioritize users.

Step-4) Calculate Allocated Data Rate to user by applying new algorithm.

Step-5) Calculate throughput.

Step-6) Evaluate Performance.

\section{CONCLUSION}

Different types of scheduling algorithms are discussed in this paper. This paper also performs comparative analysis between different resource allocation algorithm and correspondingly designed a new technique named ENGT that is termed as extended $\mathrm{Na}$ Gaunn Technique whose main function is to provide proper resource distribution on time and frequency basis and provide maximum throughput. Authors consider input of rate requirement of users and maximum CQI matrix. Priority algorithm helps to achieve a maximum rate of throughput. On the basis of that prioritization available resource blocks are allocated to users and allocated rate of each user and throughput of system is calculated. Hence, uniform allocation by priority is better in terms of allocated rate. 


\section{REFERENCES}

[1] Aderemi A. Atayero, "An Introduction to LTE". 3GPP LTE Encyclopedia. Retrieved December 3, 2010.

[2] M. Faulkner,"Long Term Evolution (LTE): A Technical Overview". Motorola.Retrieved July 3, 2010.

[3] GPP. UTRA-UTRAN long term evolution (LTE) and 3GPP system architecture evolution (SAE).http://www.3gpp.org/article/lte, 2010.

[4] Mohsen M. Tantawy, Adly S. Tag Eldien, and RokaiaMounirZaki 1, "A Novel Cross-Layer Scheduling Algorithm for Long Term-Evolution (LTE) Wireless System", Canadian Journal on Multimedia and Wireless Networks, Vol. 2, No. 4, December 2011. http://en.wikipedia.org/wiki/matlab.

[6] Raymond Kwan and Cyril Leung, “A Survey of Scheduling and Interference Mitigation in LTE”, Journal of Electrical and Computer Engineering Volume 2010, Department of Electrical and Computer Engineering,
University of British Columbia, 2332 Main Mall, Vancouver.

[7] Y. Su and L. Ming, "Backhaul Resource Allocation in LTE-Advanced Relaying Systems", IEEE Wireless Communications and Networking Conference: MAC and Cross-Layer Design, pp- 1207-1211, 2012.

Honghai Zhang, Narayan Prasad and SampathRangarajan "MIMO Downlink Scheduling in LTEsystems" in 2012, IEEE.

[9] Q. Bai, N. Passas, J. A. Nossek, "Scheduling and resource allocation in OFDM and FBMC systems: An interactive approach and performance comparison”, 2010 European Wireless Conference, April 2010.

[10] Anu Mittal, Naveen Sharma, "Various Techniques for scheduling in LTE - A Review", International journal of Emerging Technologies in computational and applied sciences, 2016

[11] SinhChuong Nguyen, KumbesanSandrasegaran and Faisal Mohd. Jamal Madani "Modeling and Simulation of Packet Scheduling in the Downlink LTE- Advanced”, IEEE, 2011.

Na Gaun, Lin Tian and Gang Sun "Qos Guaranteed Resource block allocation algorithm for LTE System”, IEEE, 2011. 